\title{
Effect of the volumetric dimensions of a complete arch on the accuracy of scanners
}

\author{
Min-Kyu Kim ${ }^{1+}$, KeunBaDa Son ${ }^{2,3+}$, Beom-Young Yu ${ }^{2,3}$, Kyu-Bok Lee ${ }^{1,2,3 *}$ \\ ${ }^{1}$ Department of Dental Science, Graduate School, Kyungpook National University, Daegu, Republic of Korea \\ ${ }^{2}$ Advanced Dental Device Development Institute (A3DI), Kyungpook National University, Daegu, Republic of Korea \\ ${ }^{3}$ Department of Prosthodontics, School of Dentistry, Kyungpook National University, Daegu, Republic of Korea
}

\begin{abstract}
PURPOSE. The present study aimed to evaluate the accuracy of a desktop scanner and intraoral scanners based on the volumetric dimensions of a complete arch. MATERIALS AND METHODS. Seven reference models were fabricated based on the volumetric dimensions of complete arch $(70 \%, 80 \%, 90 \%, 100 \%, 110 \%, 120 \%$, and 130\%). The reference models were digitized using an industrial scanner (Solutionix C500; MEDIT) for the fabrication of a computer-aided design (CAD) reference model (CRM). The reference models were digitized using three intraoral scanners (CS3600, Trios3, and i500) and one desktop scanner (E1) to fabricate a CAD test model (CTM). CRM and CTM were then superimposed using inspection software, and 3D analysis was conducted. For statistical analysis, one-way analysis of variance was used to verify the difference in accuracy based on the volumetric dimensions of the complete arch and the accuracy based on the scanners, and the differences among the groups were analyzed using the Tukey HSD test as a post-hoc test $(\alpha=.05)$. RESULTS. The three different scanners showed a significant difference in accuracy based on the volumetric dimensions of the complete arch $(P<.05)$, but the desktop scanner did not show a significant difference in accuracy based on the volumetric dimensions of the complete arch $(P=.808)$. CONCLUSION. The accuracy of the intraoral scanners was dependent on the volumetric dimensions of the complete arch, but the volumetric dimensions of the complete arch had no effect on the accuracy of the desktop scanner. Additionally, depending on the type of intraoral scanners, the accuracy differed according to the volumetric dimensions of the complete arch. [J Adv Prosthodont 2020;12:361-8]
\end{abstract}

KEYWORDS: Intraoral scanner; Accuracy; Complete arch; Dimension

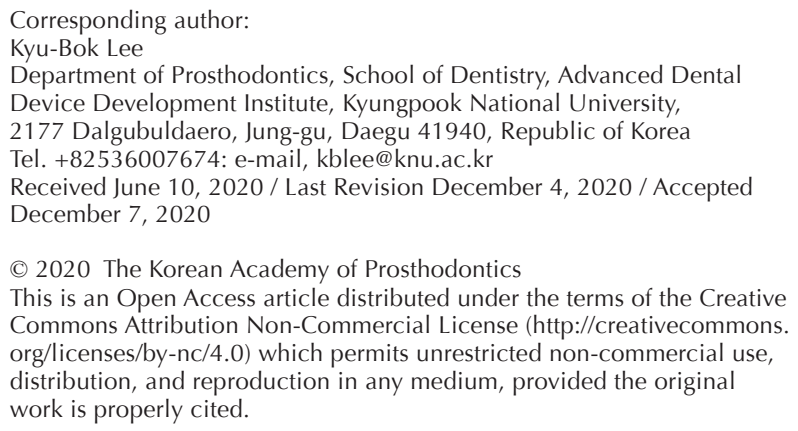

tMin-Kyu Kim and KeunBaDa Son contributed equally to this study (co-first author).

This work was supported by the Industrial Strategic Technology Development Program (10062635, New hybrid milling machine with a resolution of less than $10 \mu \mathrm{m}$ development, using open CAD/CAM S/W integrated platforms for a 1 day prosthetic treatment of 3-D smart medical care system) funded by the Ministry of Trade, Industry \& Energy (Korea); and Korea Institute for Advancement of Technology through the National Innovation Cluster R\&D program (P0006691).

\section{INTRODUCTION}

With the introduction of dental computer-aided design and computer-aided manufacturing (CAD-CAM), integrating digital technology into the conventional workflow, which had previously been dependent on the operator's experience and skill, has become possible. ${ }^{1-5}$ In the 1970 s, digital technology has been applied in clinical dentistry. ${ }^{5}$ The first-proposed digital workflow was a partially digital workflow accompanied by impression taking and definitive model fabrication. ${ }^{3,6}$ Subsequently, the development of an intraoral scanner in the 1980s has enabled a fully digital workflow. ${ }^{3,6}$

The first step of the digital workflow is to obtain a virtual model of the patient. ${ }^{7,8}$ The accuracy of the scan has a big impact on the fit of the dental removable and fixed prostheses. ${ }^{7,8}$ Factors such as ambient light, the humidity of the indoor and oral cavity, and the operator's proficiency affect the accuracy of the scan during the creation of the virtual models using either a desktop scanner or an intraoral 
scanner. ${ }^{7-11}$ Additionally, studies have found a significant difference in the accuracy of scanning based on scanner types and scan strategies. ${ }^{12-15}$

In contrast to a desktop scanner, an intraoral scanner obtains information about the oral cavity through a small scan tip in the limited space of the oral cavity. ${ }^{16,17}$ Hence, areas scanned only once are limited when using the intraoral scanner, and the virtual model is completed by stitching and aligning partially scanned areas. ${ }^{18-20}$ Accordingly, with an intraoral scanner, the distortion of the dental arch occurs at the tooth from which the scanning begins. ${ }^{3}$ Consequently, studies are divided into those that recommend the scanning range of the complete arch for fixed prostheses in the intraoral scanner ${ }^{21-23}$ and those that do not. ${ }^{2-4}$ Conversely, because the desktop scanner scans the complete arch simultaneously and completes the virtual model via stitching and alignment, little distortion occurs. ${ }^{3}$

The accuracy of scanners has been evaluated in various ways. ${ }^{24-26}$ Several studies have evaluated scanner accuracy using the 3-dimensional (3D) overlapping method, ${ }^{27-34}$ in which a virtual model obtained by a high-precision industrial scanner serves as the standard for evaluation, and the difference in the average distance between the two virtual models is calculated by overlapping the standard model with that of the virtual model obtained using a scanner to be evaluated. ${ }^{27-30}$ This method has been used by several studies as the standard evaluation of scanner accuracy. ${ }^{27-30}$ Additionally, previous studies recommended a scanner accuracy of $<100$ $\mu \mathrm{m}$ for fixed prostheses. ${ }^{3,4,16}$ However, no studies have verified the scanning accuracy required for clinical use.

Several studies that evaluated the accuracy of scanners did not consider the volumetric dimension of the complete arch. ${ }^{2-4}$ The preceding studies did not consider the volumetric dimension of the complete arch and did not evaluate the impact of the volumetric dimension. ${ }^{21-23}$ However, when obtaining a virtual model of the complete arch using an intraoral scanner, the distortion of the dental arch occurs, and the accuracy of the scan may vary depending on the volumetric dimension of the complete arch.

In the present study, we aimed to evaluate the accuracy of both a desktop scanner and intraoral scanners based on the volumetric dimensions of a complete arch. The first null hypothesis is that there would be no difference in accuracy between the desktop scanner and the intraoral scanner based on the volumetric dimensions of the complete arch. The second null hypothesis is that there would be no difference in accuracy among the scanners at each volumetric dimension of the complete arch.

\section{MATERIALS AND METHODS}

A dental typodont model (ANKA-4 V CER; Frasaco, Tettnang, Germany) was scanned with an industrial scanner (Solutionix C500; MEDIT, Seoul, Republic of Korea) to fabricate a virtual model. The volumetric dimensions of the complete arch of the obtained virtual model were adjusted using 3D printer software (ZENITH Software; Dentis,
Daegu, Republic of Korea). Based on all of the obtained virtual models, a total of seven virtual models were fabricated by changing the volumetric dimensions by $10 \%(70 \%$, $80 \%, 90 \%, 100 \%, 110 \%, 120 \%$, and $130 \%$ ). The seven fabricated virtual models were printed out using a $3 \mathrm{D}$ printer (ZENITH U; Dentis) via a stereolithography apparatus method. With the models printed out as recommended by the manufacturer, the remaining resin was removed using $83 \%$ ethanol and light-cured for $10 \mathrm{~min}$ in an ultraviolet light-curing device (CURE DEN; Dentis). Additionally, to prevent light reflex on the surface of the resin models, they were duplicated as stone models. The duplicated stone models were set as the reference models.

Using an industrial scanner (Solutionix C500) with a high resolution of $2 \times 5$ megapixels, an accuracy below 10 $\mu \mathrm{m}$, and a blue light-emitting diode, seven reference models were scanned. The industrial 3D scanner was calibrated before scanning, and according to the manufacturer's instructions, the seven reference models were scanned precisely. Additionally, the scanned virtual model was designated as a CRM file (Fig. 1). The results of the measurements of the width and the length of the complete arch in the CRM file, referring to the measurement method of the previous study, are shown in Table $1 .^{31}$

In the present study, we used three different scanners (CS3600 [Carestream Dental, Atlanta, GA, USA], Trios3 [3Shape, Copenhagen, Denmark], and i500 [MEDIT]) and one desktop scanner (E1 [3Shape]) to fabricate a CTM of the reference model (Table 2). The three different intraoral scanners were calibrated before scanning, and according to the manufacturer's instructions, the seven reference models were scanned precisely. Because the accuracy of the intraoral scanners might vary depending on the order of complete arch scanning, the present study applied the scanning sequence associated with the best accuracy as reported by a previous study (Fig. 2). ${ }^{35}$ Additionally, according to ISO12836, scanners were set at an ambient temperature of

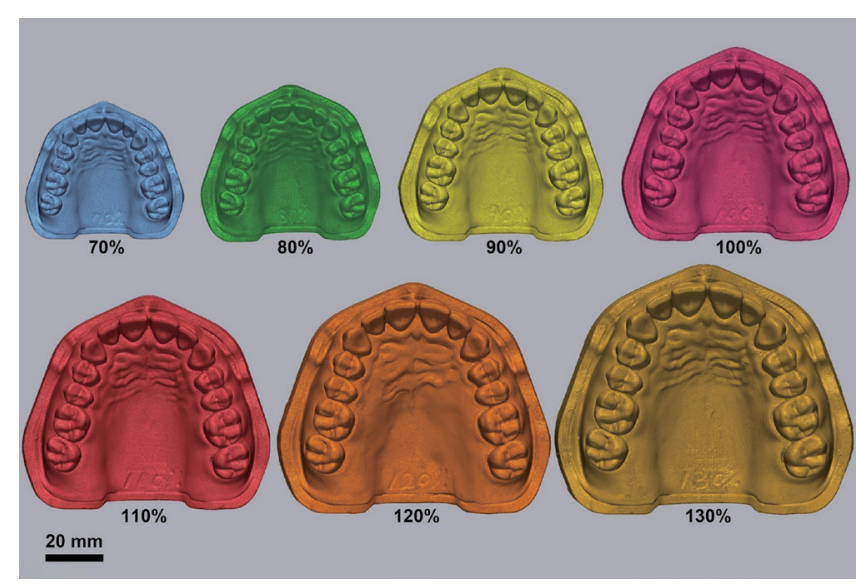

Fig. 1. CAD reference models according to the volumetric dimensions of the complete arch. 
Table 1. Complete arch width and length of CAD reference models according to volumetric dimensions of the complete arch

\begin{tabular}{cccc}
\hline $\begin{array}{c}\text { Volumetric } \\
\text { dimension of the } \\
\text { complete arch (\%) }\end{array}$ & $\begin{array}{c}\text { Intercanine } \\
\text { width }^{*}\end{array}$ & $\begin{array}{c}\text { Intermolar } \\
\text { width* } \\
\text { Distance (mm) }\end{array}$ & Arch length ${ }^{\star *}$ \\
\hline 70 & 27.05 & 38.45 & 52.54 \\
80 & 30.78 & 43.29 & 60.88 \\
90 & 34.45 & 49 & 67.96 \\
100 & 38.03 & 54.62 & 75.82 \\
110 & 41.93 & 59.54 & 82.58 \\
120 & 46.03 & 65.94 & 89.8 \\
130 & 50.58 & 71.09 & 96.78 \\
\hline
\end{tabular}

* Arch width measurements: intercanine width, the distance between the cusp tips of the canines; intermolar width, the distance between the mesiobuccal cusp tips of the first permanent molars.

${ }^{*}$ Arch length measurements: anterior arch length, the distance between the contact point of the central incisors and contact point between the canine and first premolar; posterior arch length, the distance between the contact point of the canine and the first premolar and the contact point between the second premolar and the first permanent molar; total arch length, the sum of the anterior and posterior arch lengths.

$23^{\circ} \mathrm{C} \pm 2{ }^{\circ} \mathrm{C}$ during scanning, ${ }^{36}$ and a single operator (M.K.) skilled in using each intraoral scanner conducted the scanning 17 times $(\mathrm{N}=17)$. Scanning accuracy was evaluated through the five pilot experiments prior to the initiation of the experimental condition and via power analysis software (G*Power 3.1.9.2; Heinrich-Heine-Universität Düsseldorf, Germany). In the present study, it has been found that 17 scans were necessary (actual power $=99.76 \%$, power $=$ $99.7 \%, \alpha=0.05$ ). Additionally, for the 3D analysis, the data were exported into a standard tessellation language file.

First, to conduct the 3D analysis of the displacement of the teeth using only the 3D analysis program (Geomagic Control X 2018.0.0; 3D Systems, Cary, NC, USA), the teeth were only segmented by excluding the gingiva from the CRM file (Fig. 3A). After preparing the CRM file, the CTM file was retrieved (Fig. 3B), and the initial alignment was conducted. Additionally, in the CRM file, only the segmented teeth were designated as the area for conducting the best fit alignment (Fig. 3C). At this time, the sampling ratio was designated at $100 \%$.

The 3D difference between the CRM file and the CTM file was calculated for all data points of the segmented teeth. Data points were calculated using the root mean square (RMS) with the following formula: $: 30$

$$
R M S=\frac{1}{\sqrt{n}} \cdot \sqrt{\sum_{i=1}^{n}\left(X_{1, i}-X_{2, i}\right)^{2}}
$$
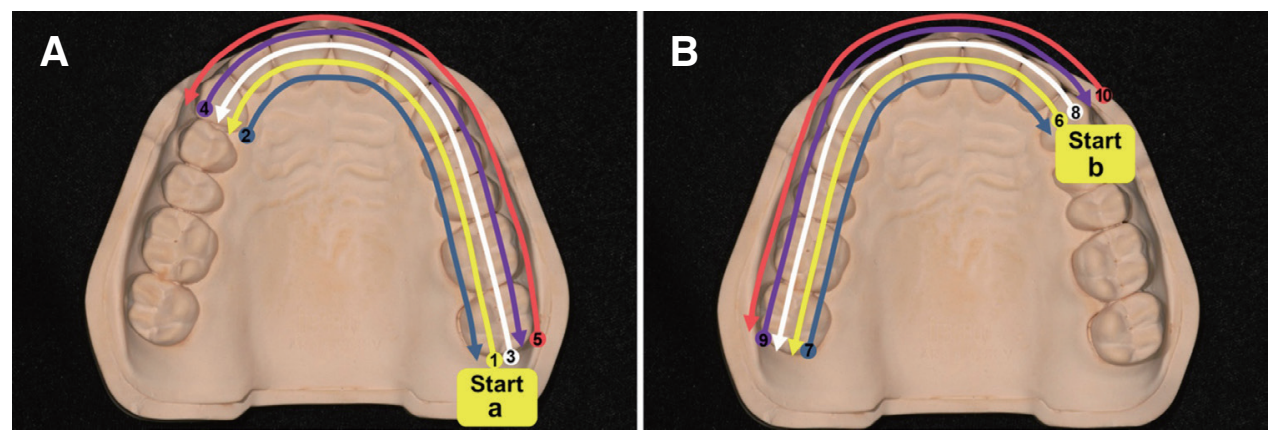

Fig. 2. Strategies of complete arch scanning. (A) First scanning procedure from "Start a" to opposite canine (1 to 5), (B) Second scanning procedure from "Start $b$ " to opposite second molar (6 to 10).

Table 2. Intraoral scanners tested

\begin{tabular}{cccc}
\hline System & Scanner technology & Light source & Acquisition method \\
\hline CS3600 & Confocal microscopy & Light & Video sequence \\
Trios3 & Confocal microscopy & Light & Video sequence \\
i500 & Triangulation technology & Light & Video sequence \\
\hline
\end{tabular}



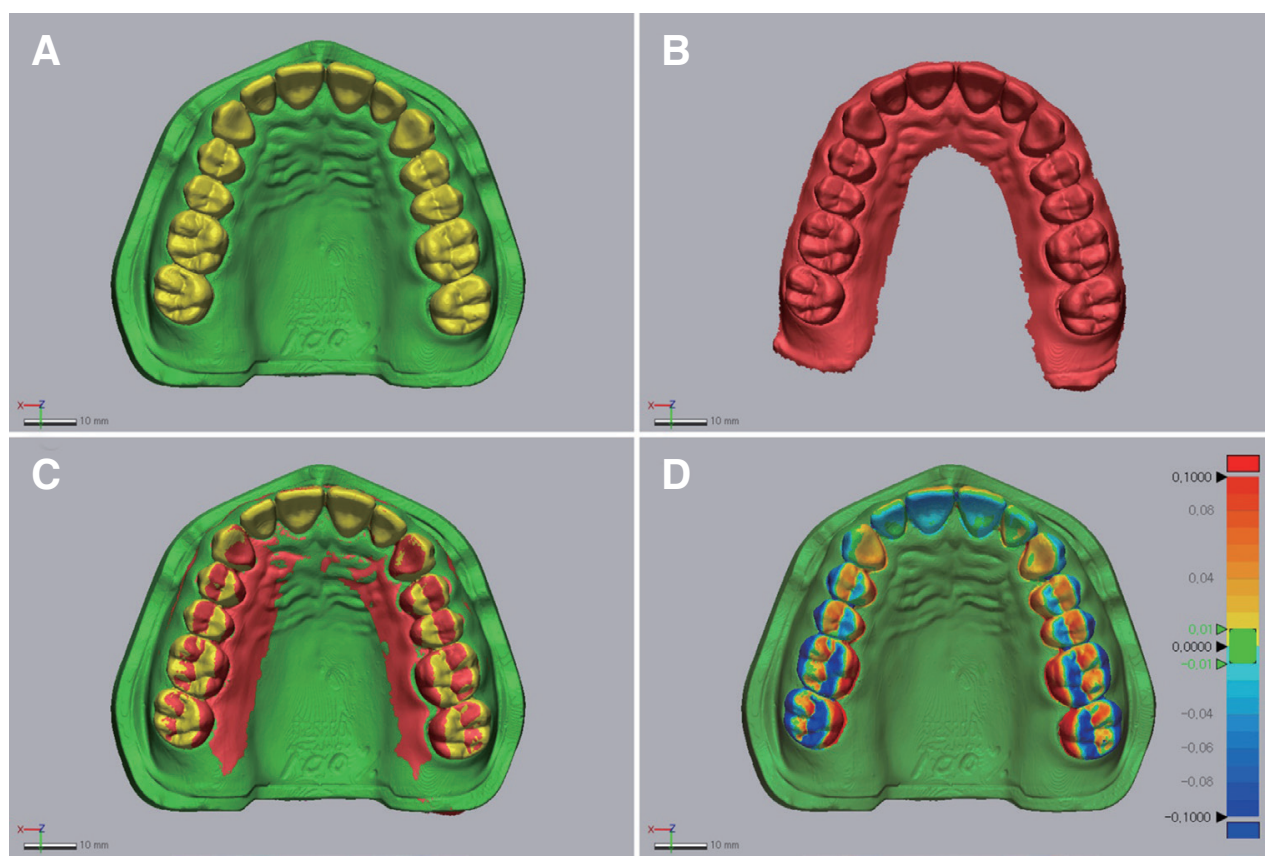

Fig. 3. Three-dimensional analysis procedure. (A) Tooth segmentation of CAD reference model (CRM), (B) CAD test model (CTM), (C) Superimposition of CRM and CTM, (D) Color difference map by 3D analysis.

where $X_{1, i}$ is the measurement point of $i$ in the CRM, $X_{2, i}$ is the measurement point of $i$ in the CTM, and $n$ refers to the number of all points measured in each analysis. The RMS shows the deviation between the two sets of data. Thus, a low RMS indicates a high 3D agreement of the superimposed data. Additionally, the 3D comparison was shown by a color difference map and a 3D displacement color range of $\pm 100 \mu \mathrm{m}$ (20 color segments). A color range with a tolerance of $\pm 10 \mu \mathrm{m}$ was designated as green (Fig. 3D), indicating a very high agreement between the CRM and the CTM.

All data were analyzed using statistical analysis software (SPSS Software 25.0; IBM, Armonk, NY, USA) ( $\alpha=.05)$. First, using the Shapiro-Wilk test, the normal distribution of data was examined. A normal distribution was identified, and the equality of the distribution was evaluated using the Levene test. To evaluate the differences in accuracy based on the volumetric dimension of the complete arch and accuracy based on the scanner type, one-way analysis of variance (ANOVA) was used, and the difference among the groups was analyzed using the Tukey HSD test as a posthoc test. Lastly, to evaluate the interaction effect between the scanner factor and the volumetric dimension of the complete arch factor, an analysis was conducted using twoway ANOVA.

\section{RESULTS}

There was a significant difference in accuracy according to scanner types based on all volumetric dimensions of the complete arch $(P<.001)$ (Fig. 4; Table 3). Additionally, depending on the volumetric dimension of the complete arch, the three intraoral scanner types (CS3600, Trios3, and i500) showed significant differences in accuracy $(P<.05)$ (Fig. 4; Table 3). Meanwhile, there was no significant difference in accuracy based on the volumetric dimension of the complete arch in the desktop scanner $(P=.808)$ (Fig. 4; Table 3).

CS3600 showed the best accuracy at 70\% (30.1 \pm 1.5 $\mu \mathrm{m})$, whereas it showed the worst accuracy at 130\% (65.4 \pm $5.8 \mu \mathrm{m}$ ) (Table 3). Trios3 showed the best accuracy at $70 \%$ $(22.3 \pm 2.2 \mu \mathrm{m})$, whereas it showed the worst accuracy at $120 \%(44.2 \pm 6.4 \mu \mathrm{m})$ (Table 3). i500 showed the best accuracy at $100 \%(33.8 \pm 9.6 \mu \mathrm{m})$, whereas it showed the worst accuracy at $70 \%(64.9 \pm 19.2 \mu \mathrm{m})$ and $120 \%(63.3 \pm$ $11.6 \mu \mathrm{m})($ Table 3).

The present study found that the scanner type and the volumetric dimension of a complete arch had an interaction effect $(P<.001)$ and that these two factors affected the accuracy (Table 3).

The color difference map showed a tendency similar to the result shown in Table 3 (Fig. 5). In the desktop scanner, green areas of $10 \mu \mathrm{m}$ were mostly observed in all volumetric dimensions of the complete arch (Fig. 5A). Meanwhile, there were larger errors in the intraoral scanners than in the desktop scanner, and the displacement aspect differed depending on the intraoral scanner (Fig. 5). In CS3600 and Trios3, the largest green area of $10 \mu \mathrm{m}$ was observed at $70 \%$ (Fig. 5B and C), whereas with the i500, the largest green area was observed at 100\% (Fig. 5D). 


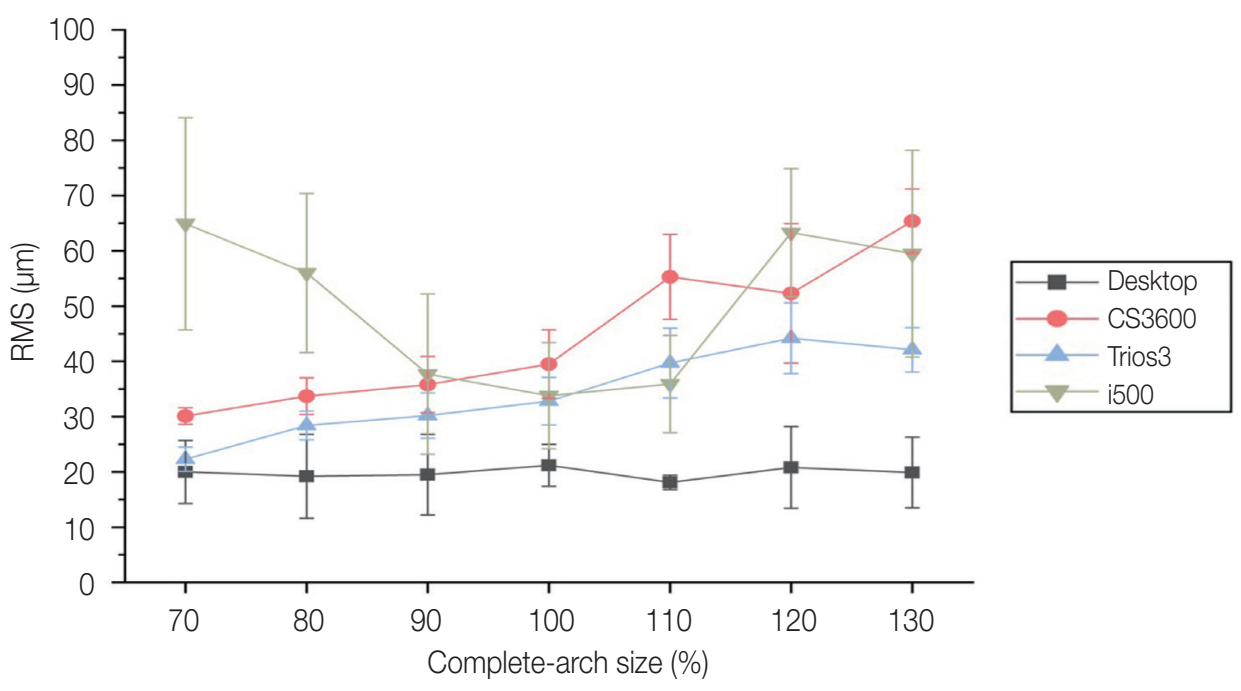

Fig. 4. Comparison of RMS values according to volumetric dimensions of complete arch and scanner type.

Table 3. Comparison of RMS values according to volumetric dimensions of a complete arch and a scanner type

\begin{tabular}{|c|c|c|c|c|c|c|}
\hline \multirow{2}{*}{$\begin{array}{l}\text { Volumetric dimension of } \\
\text { a complete arch (\%) }\end{array}$} & Desktop & CS3600 & Trios3 & $i 500$ & \multirow{2}{*}{$\mathrm{F}$} & \multirow{2}{*}{$P$} \\
\hline & \multicolumn{4}{|c|}{$\mathrm{RMS}(\mathrm{mean} \pm \mathrm{SD}, \mu \mathrm{m})$} & & \\
\hline 70 & $20 \pm 5.7^{\mathrm{A}}$ & $30.1 \pm 1.5^{\mathrm{aB}}$ & $22.3 \pm 2.2^{\mathrm{aAB}}$ & $64.9 \pm 19.2^{\mathrm{abc}}$ & 71.72 & $<.001^{*}$ \\
\hline 80 & $19.2 \pm 7.6^{A}$ & $33.7 \pm 3.3^{\mathrm{abB}}$ & $28.4 \pm 2.6^{\mathrm{bB}}$ & $56 \pm 14.4^{\mathrm{abc}}$ & 58.63 & $<.001^{*}$ \\
\hline 90 & $19.5 \pm 7.3^{\mathrm{A}}$ & $35.8 \pm 5.1^{\mathrm{abB}}$ & $30.2 \pm 4.1^{\mathrm{bB}}$ & $37.7 \pm 14.5^{\mathrm{abB}}$ & 14.6 & $<.001^{*}$ \\
\hline 100 & $21.2 \pm 3.8^{A}$ & $39.5 \pm 6.2^{\mathrm{bB}}$ & $32.8 \pm 4.3^{b c}$ & $33.8 \pm 9.6^{\mathrm{aBC}}$ & 24.19 & $<.001^{*}$ \\
\hline 110 & $18.1 \pm 1.3^{\mathrm{A}}$ & $55.3 \pm 7.7^{\mathrm{cB}}$ & $39.7 \pm 6.3^{\mathrm{CB}}$ & $35.9 \pm 8.8^{\mathrm{abc}}$ & 87.62 & $<.001^{*}$ \\
\hline 120 & $20.8 \pm 7.4^{\mathrm{A}}$ & $52.3 \pm 12.6^{\mathrm{CB}}$ & $44.2 \pm 6.4^{\mathrm{CB}}$ & $63.3 \pm 11.6^{\mathrm{abc}}$ & 55.91 & $<.001^{*}$ \\
\hline 130 & $19.9 \pm 6.4^{A}$ & $65.4 \pm 5.8^{\mathrm{dAB}}$ & $42.1 \pm 4^{\mathrm{CAB}}$ & $59.5 \pm 18.7^{\mathrm{bB}}$ & 2.88 & $.042^{*}$ \\
\hline $\mathrm{F}$ & .5 & 61.92 & 52.79 & 2.27 & & \\
\hline$P$ & $.808^{*}$ & $<.001^{\star}$ & $<.001^{\star}$ & $.041^{*}$ & & \\
\hline $\begin{array}{l}\text { Scanner type * } \\
\text { Volumetric dimension type }\end{array}$ & $.001^{\star *}$ & & & & & \\
\hline
\end{tabular}

* Significant by one-way ANOVA; $P<.05$. Different letters (uppercase: row; lowercase: column) indicate significant differences by the Tukey honestly significant difference test $(P<.05)$.

** Significant by two-way ANOVA; $P<.05$.

\section{DISCUSSION}

In the present study, we evaluated the accuracy of the scanners according to the volumetric dimension of the complete arch. We found a significant impact on accuracy based on the volumetric dimension of the complete arch for the intraoral scanners. Additionally, the accuracy was affected by the scanner at each volumetric dimension of the complete arch. Thus, except for desktop scanners $(P=.808)$, all hypotheses in the present study were rejected $(P<.001)$.

Several previous studies have reported that desktop scanners were more accurate than intraoral scanners. ${ }^{3-5}$ Likewise, in the present study, the same result was shown in which the desktop scanner was more accurate. In previous studies, differences between desktop scanners and intraoral scanners were compared in one study model, ${ }^{3,4}$ but in the present study, significant differences were observed with the size of the arch as a variable. When comparing the desktop scanner and the intraoral scanner, the size of the arch did not affect the accuracy of the desktop scanner, but the size of the arch had a significant effect on the intraoral scanner.

Many previous studies have evaluated the accuracy of intraoral scanners in a complete arch scan..$^{2-4}$ Braian and Wennerberg reported that the accuracy of intraoral scanners (Omnicam, CS3600, Emerald, iTero, and Trios3) ranged from 6 to $150 \mu \mathrm{m}$ in a complete arch scan. ${ }^{2}$ Ender et al. reported an accuracy of intraoral scanners (Bluecam, Omnicam, iTero, Lava COS, True Definition, and Trios2) that ranged from 12.3 to $167.2 \mu \mathrm{m} .{ }^{4}$ In the present study, the accuracy of the intraoral scanners at a size of $100 \%$ in a 
A

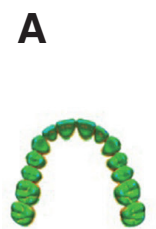

$70 \%$

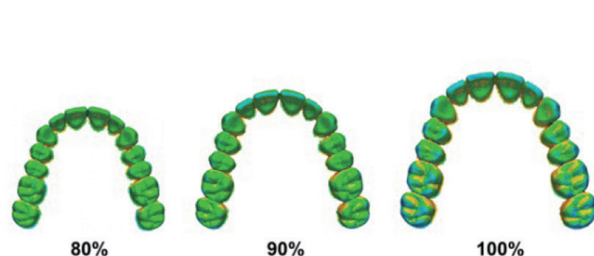

$80 \%$

$90 \%$

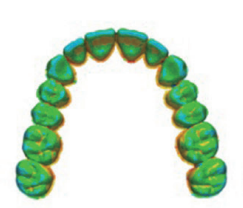

$110 \%$

$20 \mathrm{~mm}$

C

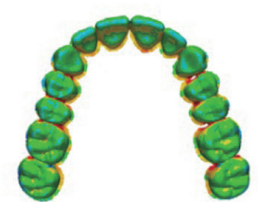

$120 \%$

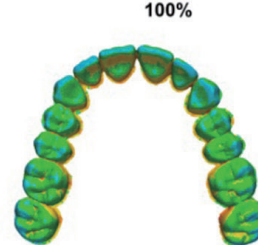

$130 \%$

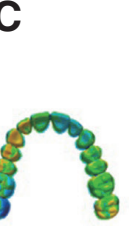

$70 \%$

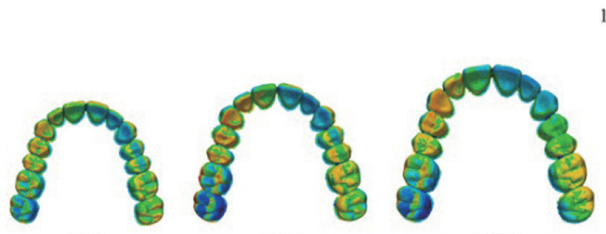

$80 \%$

$90 \%$

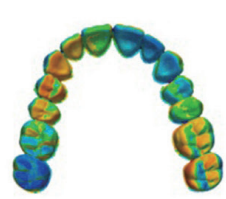

$110 \%$

$20 \mathrm{~mm}$

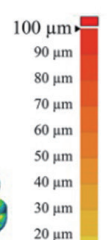

B

B

$\mu \mathrm{m}$

$10 \mu \mathrm{mo}$

$-10 \mu \mathrm{mm}$

$-30 \mu \mathrm{m}$
$-40 \mu \mathrm{m}$

$-50 \mu \mathrm{m}$

$.60 \mu \mathrm{m}$

$.70 \mu \mathrm{m}$

$-80 \mu \mathrm{m}$
$-90 \mu \mathrm{m}$

$-100 \mu \mathrm{m}$

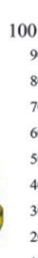

$100 \mu \mathrm{m}$
$90 \mu \mathrm{m}$
$80 \mu \mathrm{m}$
$70 \mu \mathrm{m}$
$60 \mu \mathrm{m}$
$50 \mu \mathrm{m}$
$40 \mu \mathrm{m}$
$30 \mu \mathrm{m}$
$20 \mu \mathrm{m}$
$10 \mu \mathrm{m}$

$90 \mu \mathrm{m}$
$80 \mu \mathrm{m}$
$70 \mu \mathrm{m}$
$60 \mu \mathrm{m}$
$50 \mu \mathrm{m}$
$40 \mu \mathrm{m}$
$30 \mu \mathrm{m}$
$20 \mu \mathrm{m}$

D

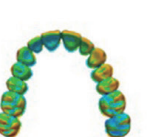

$70 \%$

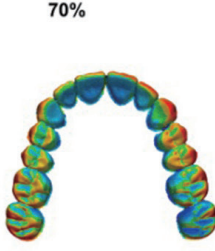

$\mathrm{mm}$

\begin{abstract}
.
\end{abstract}

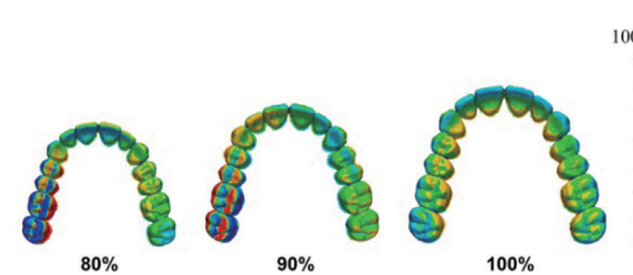

$70 \%$

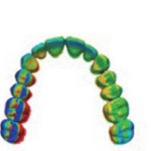

$80 \%$

${ }_{80 \%}{ }_{90 \%}{ }_{100 \%}$

$100 \%$. $\begin{gathered}30 \mu \mathrm{m} \\ 20 \mu \mathrm{m} \\ 10 \mu \mathrm{mm}\end{gathered}$

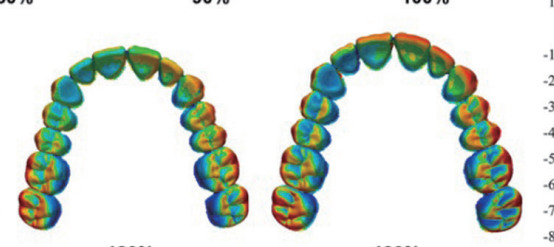

$130 \%$

$90 \%$

$100 \%$

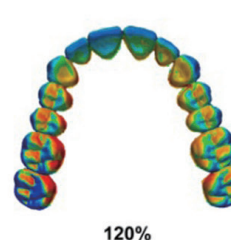

$120 \%$
$20 \mathrm{~mm}$

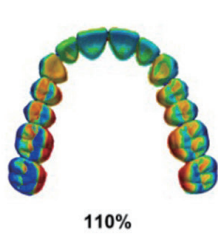

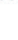
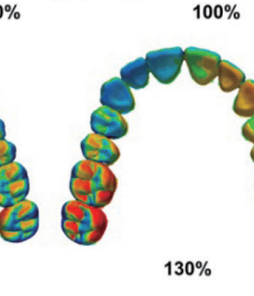

$130 \%$
$\Omega$

Fig. 5. Comparison of color difference map according to volumetric dimensions of complete arch and scanner type. (A) Desktop, (B) CS3600, (C) Trios3, (D) i500.

complete arch scan (CS3600, Trios3, and i500) was in a small range of 32.8 to $39.5 \mu \mathrm{m}$ (Table 3 ). The difference among the results of the present study and the findings of preceding studies is due to the difference in the method of obtaining CRMs, the 3D analysis method, and the intraoral scanner type. ${ }^{27-30}$ Additionally, the difference in the acquisition of CRMs can be due to the difference in the specifications of the scanners used in most studies. ${ }^{27-30}$ The accuracy and reliability of the scanner used for obtaining CRMs are the most essential factors in the accuracy evaluation. Accordingly, the present study ensured the accuracy and reliability by using high-definition industrial scanners by obtaining CRMs from experts in the manufacturer of industrial 3D scanner. Moreover, in the 3D analysis method, since various $3 \mathrm{D}$ analysis programs are aligned using different protocols, the result of the $3 \mathrm{D}$ analysis may vary. 3,30 Thus, the present study used the Geomagic 3D analysis program, which is recommended by ISO $12836 .{ }^{3}$ Lastly, the accuracy of the scanning may vary depending on the intraoral scanner type. ${ }^{19-27}$ Manufacturers of intraoral scanners have recently updated their products to enhance the accuracy of complete arch. Therefore, an objective comparison of previous studies is challenging. The evaluation should be made while considering these factors, and additional studies on the most recent intraoral scanners are needed.

The most essential process in the accuracy evaluation in this study is the fabrication of various volumetric dimensions of a complete arch. Thus, this study fabricated seven CRMs, increasing or decreasing by $10 \%$ the size of the dental typodont often used as the standard for evaluation based on the dimension of $100 \%$ (Fig. 1). The dental arch dimension was measured in seven CRMs (Table 1); however, the measurements cannot represent the dental arch dimensions in all ages, nationalities, and races. Thus, conducting a further study of the relationship between the dental arch dimension and the accuracy by further subdividing it by age, nationality, and race is important.

In the present study, the left and right asymmetric results of $3 \mathrm{D}$ accuracy results shown in Fig. 5B, 5C, and 5D can be attributed to the scanning sequence of the intraoral scanner. The tip of the intraoral scanner can show asymmetric results depending on the scanning sequence due to the small tip that can scan one or two teeth at a time. A previous study has reported that the further away from the scan start point, the higher the inaccuracy of the scan. ${ }^{3}$ In the present study, the scan sequence showing the best accu- 
racy in the previous study was applied to reduce the error due to the scan sequence, ${ }^{35}$ and all models were duplicated with dental stone to prevent errors due to $3 \mathrm{D}$ printing. Further research is required to accurately identify the area of the dental arch where the difference in accuracy based on the dimension differences can be determined.

We used the model of identical figures in the present study only to compare the accuracy with varying sizes. Another previous study has concluded that accuracy can vary significantly depending on the shapes of the arch and the teeth of different patients. ${ }^{37}$ Therefore, in the present study, using the model of identical figures, the accuracy of the desktop and intraoral scanners has been evaluated.

Because an intraoral scanner acquires information regarding the oral cavity through a small scan tip in the limited space in the oral cavity, unlike a desktop scanner, ${ }^{16,17}$ the present study expected that the accuracy of the intraoral scanners would be the best in the volumetric dimension of a complete arch of $70 \%$. The CS3600 and Trio3 showed the best accuracy at a volumetric dimension of a complete arch of $70 \%$, whereas the $i 500$ showed the best accuracy at a volumetric dimension of a complete arch of $100 \%$ (Table 3). Additionally, the aspect of accuracy differed depending on the dental arch dimension in each of the intraoral scanners used in this study. The larger the volumetric dimension of the complete arch, the poorer the accuracy became in CS3600 and Trio3, whereas the accuracy became poorer when it exceeded $100 \%$ in i500 (Table 3). We verified that the dental arch dimension affects the accuracy of the intraoral scanners. The authors of the present study can guess why the 1500 shows better accuracy in the 100\% model rather than the smaller $70 \%$ model as the difference in the alignment algorithm. Post-processing of scan data via software may vary from manufacturer to manufacturer, and tooth size may have an effect. Further research is needed on the effect of tooth size on alignment.

The present study produced seven models that increased and decreased by $10 \%$ based on the $100 \%$ volumetric dimension. All these models were scanned 17 times with four different scanners, and a total of 476 complete arch scans were conducted. Indeed, we aimed to confirm various changes through changes in the increase and decrease of the volumetric dimension a little more, but we were unable to do so because there were a large number of scans and we had limited time. Therefore, further studies are required in the range of more subdivided volumetric dimensions.

The reason that it was produced based on the volumetric dimension in the present study is that the software increased and decreased by $10 \%$ based on the $100 \%$ volumetric dimension is more convenient. Additional research is required through the model manufactured based on the linear dimension.

This study had a few limitations. First, the method of the present study did not reflect the conditions that might occur in the oral cavity in an in vitro experiment (i.e., humid environment and the impact of ambient light). ${ }^{7,8,11}$ Thus, an additional study is required to evaluate the scanning accuracy while applying the conditions that might occur in the oral cavity.

\section{CONCLUSION}

Within the limitations of this in vitro study, the following conclusions were drawn:

The accuracy of the intraoral scanner varied depending on the volumetric dimensions of the complete arch, although the accuracy of the desktop scanner was not affected.

The aspect of accuracy based on the volumetric dimensions of the complete arch differed depending on the intraoral scanner type.

CS3600 and Trios3 intraoral scanners showed the best accuracy at the volumetric dimension of the complete arch of $70 \%$; the larger the volumetric dimension of the complete arch, the poorer the accuracy.

The accuracy was the best at a volumetric dimension of the complete arch of $100 \%$ using the i500 intraoral scanner, and the accuracy became poorer when it exceeded 100\%.

The accuracy of the intraoral scanner differed depending on the volumetric dimension of the complete arch; however, the accuracy of scanning was within the clinically acceptable range in all of the intraoral scanners $(<100 \mu \mathrm{m})$.

\section{ACKNOWLEDGMENTS}

The authors thank the researchers in the Advanced Dental Device Development Institute (A3DI), Kyungpook National University, for their time and contributions to the study. The authors thank the Dr. Dong Keun Chung and staff in the Segyero dental hospital for their support to the study.

\section{ORCID}

Min-Kyu Kim https://orcid.org/0000-0002-3008-2145

KeunBaDa Son https://orcid.org/0000-0002-3177-8005

Beom-Young Yu https://orcid.org/0000-0003-1070-3386

Kyu-Bok Lee https://orcid.org/0000-0002-1838-7229

\section{REFERENCES}

1. Park JM, Kim RJ, Lee KW. Comparative reproducibility analysis of 6 intraoral scanners used on complex intracoronal preparations. J Prosthet Dent 2020;123:113-20.

2. Braian M, Wennerberg A. Trueness and precision of 5 intraoral scanners for scanning edentulous and dentate completearch mandibular casts: A comparative in vitro study. J Prosthet Dent 2019;122:129-36.

3. Park GH, Son K, Lee KB. Feasibility of using an intraoral scanner for a complete-arch digital scan. J Prosthet Dent 2019;121:803-10.

4. Ender A, Attin T, Mehl A. In vivo precision of conventional and digital methods of obtaining complete-arch dental impressions. J Prosthet Dent 2016;115:313-20.

5. Duret F, Blouin JL, Duret B. CAD-CAM in dentistry. J Am Dent Assoc 1988;117:715-20.

6. Arezoobakhsh A, Shayegh SS, Jamali Ghomi A, Hakimaneh 
SMR. Comparison of marginal and internal fit of 3-unit zirconia frameworks fabricated with CAD-CAM technology using direct and indirect digital scans. J Prosthet Dent 2020;123: 105-12.

7. Revilla-León M, Jiang P, Sadeghpour M, Piedra-Cascón W, Zandinejad A, Özcan M, Krishnamurthy VR. Intraoral digital scans-Part 1: Influence of ambient scanning light conditions on the accuracy (trueness and precision) of different intraoral scanners. J Prosthet Dent 2020;124:372-8.

8. Revilla-León M, Jiang P, Sadeghpour M, Piedra-Cascón W, Zandinejad A, Özcan M, Krishnamurthy VR. Intraoral digital scans: Part 2-influence of ambient scanning light conditions on the mesh quality of different intraoral scanners. J Prosthet Dent 2020;124:575-80.

9. Al Hamad KQ. Learning curve of intraoral scanning by prosthodontic residents. J Prosthet Dent 2020;123:277-83.

10. Lim JH, Park JM, Kim M, Heo SJ, Myung JY. Comparison of digital intraoral scanner reproducibility and image trueness considering repetitive experience. J Prosthet Dent 2018;119: 225-32.

11. Park HN, Lim YJ, Yi WJ, Han JS, Lee SP. A comparison of the accuracy of intraoral scanners using an intraoral environment simulator. J Adv Prosthodont 2018;10:58-64.

12. Michelinakis G, Apostolakis D, Tsagarakis A, Kourakis G, Pavlakis E. A comparison of accuracy of 3 intraoral scanners: A single-blinded in vitro study. J Prosthet Dent 2020;124:581-8.

13. Medina-Sotomayor P, Pascual-Moscardo A, Camps A I. Accuracy of 4 digital scanning systems on prepared teeth digitally isolated from a complete dental arch. J Prosthet Dent 2019;121:811-20.

14. Nedelcu RG, Persson AS. Scanning accuracy and precision in 4 intraoral scanners: an in vitro comparison based on 3-dimensional analysis. J Prosthet Dent. 2014;112:1461-71.

15. Nedelcu R, Olsson P, Nyström I, Thor A. Finish line distinctness and accuracy in 7 intraoral scanners versus conventional impression: an in vitro descriptive comparison. BMC Oral Health 2018;18:27.

16. Chiu A, Chen YW, Hayashi J, Sadr A. Accuracy of CAD/ CAM digital impressions with different intraoral scanner parameters. Sensors (Basel) 2020;20:1157.

17. Oh KC, Park JM, Moon HS. Effects of scanning strategy and scanner type on the accuracy of intraoral scans: A new approach for assessing the accuracy of scanned data. J Prosthodont 2020;29:518-23.

18. Koulivand S, Ghodsi S, Siadat H, Alikhasi M. A clinical comparison of digital and conventional impression techniques regarding finish line locations and impression time. J Esthet Restor Dent 2019;32:236-43.

19. Abdel-Azim T, Rogers K, Elathamna E, Zandinejad A, Metz M, Morton D. Comparison of the marginal fit of lithium disilicate crowns fabricated with CAD/CAM technology by using conventional impressions and two intraoral digital scanners. J Prosthet Dent 2015;114:554-9.

20. Sami T, Goldstein G, Vafiadis D, Absher T. An in vitro 3D evaluation of the accuracy of 4 intraoral optical scanners on a 6-implant model. J Prosthet Dent 2020;124:748-54.

21. Ender A, Zimmermann M, Mehl A. Accuracy of complete- and partial-arch impressions of actual intraoral scanning systems in vitro. Int J Comput Dent 2019;22:11-9.

22. Bilmenoglu C, Cilingir A, Geckili O, Bilhan H, Bilgin T. In vitro comparison of trueness of 10 intraoral scanners for implant-supported complete-arch fixed dental prostheses. J Prosthet Dent 2020;124:755-60.

23. Renne W, Ludlow M, Fryml J, Schurch Z, Mennito A, Kessler $\mathrm{R}$, Lauer A. Evaluation of the accuracy of 7 digital scanners: An in vitro analysis based on 3-dimensional comparisons. J Prosthet Dent 2017;118:36-42.

24. Camardella LT, Breuning H, de Vasconcellos Vilella O. Accuracy and reproducibility of measurements on plaster models and digital models created using an intraoral scanner. J Orofac Orthop 2017;78:211-20.

25. Zimmermann M, Koller C, Rumetsch M, Ender A, Mehl A. Precision of guided scanning procedures for full-arch digital impressions in vivo. J Orofac Orthop 2017;78:466-71.

26. Uhm SH, Kim JH, Jiang HB, Woo CW, Chang M, Kim KN, Bae JM, Oh S. Evaluation of the accuracy and precision of four intraoral scanners with $70 \%$ reduced inlay and four-unit bridge models of international standard. Dent Mater J 2017;36:27-34.

27. Marghalani A, Weber HP, Finkelman M, Kudara Y, El Rafie K, Papaspyridakos P. Digital versus conventional implant impressions for partially edentulous arches: An evaluation of accuracy. J Prosthet Dent 2018;119:574-9.

28. Patzelt SB, Bishti S, Stampf S, Att W. Accuracy of computeraided design/computer-aided manufacturing-generated dental casts based on intraoral scanner data. J Am Dent Assoc 2014; 145:1133-40.

29. Persson AS, Andersson M, Odén A, Sandborgh-Englund G. Computer aided analysis of digitized dental stone replicas by dental CAD/CAM technology. Dent Mater 2008;24:1123-30.

30. Kaindl K, Steipe B. Metric properties of the root-meansquare deviation of vector sets. Acta Crystallogr A 1997;53:809.

31. Bu X, Khalaf $\mathrm{K}$, Hobson RS. Dental arch dimensions in oligodontia patients. Am J Orthod Dentofacial Orthop 2008;134:76872.

32. Dung TM, Ngoc VT, Hiep NH, Khoi TD, Van Xiem V, ChuDinh T, Bac ND. Evaluation of dental arch dimensions in 12 year-old Vietnamese children-A cross-sectional study of 4565 subjects. Sci Rep 2019;9:1-7.

33. Yzquierdo-Correa C, Portocarrero-Reyes W, Carruitero MJ, Claudet-Angulo P. Dental arch width in overweight and normal-weight subjects. J Dent Res 2019;8:9-12.

34. Haque F, Alam MK. Tooth size dimension norms and sexual disparities for various populations: An overview. Int Med J 2017;24:272-4.

35. Latham J, Ludlow M, Mennito A, Kelly A, Evans Z, Renne W. Effect of scan pattern on complete-arch scans with 4 digital scanners. J Prosthet Dent 2020;123:85-95.

36. ISO 12836. Dentistry-Digitizing devices for CAD/CAM systems for indirect dental restorations-Test methods for assessing accuracy. International Standards Organization (ISO); Geneva; Switzerland, 2015. Available at: https://www.iso.org/ obp/ui/\#iso:std:iso:12836:ed-2:v1:en Accessed July 31, 2018.

37. Son K, Lee KB. Effect of tooth types on the accuracy of dental 3d scanners: An in vitro study. Materials (Basel) 2020;13:1744. 